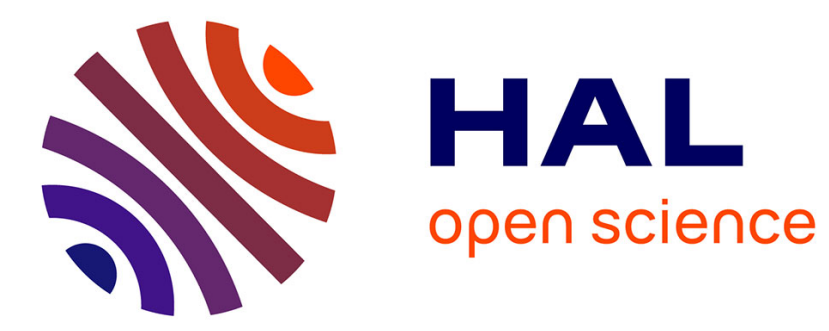

\title{
Smectic order and backbone anisotropy of a side-chain liquid crystalline polymer by Small-Angle Neutron Scattering
}

Laurence Noirez, G. Pépy, P. Keller, Ludovic Benguigui

\section{- To cite this version:}

Laurence Noirez, G. Pépy, P. Keller, Ludovic Benguigui. Smectic order and backbone anisotropy of a side-chain liquid crystalline polymer by Small-Angle Neutron Scattering. Journal de Physique II, 1991, 1 (7), pp.821-830. 10.1051/jp2:1991100 • jpa-00247558

HAL Id: jpa-00247558

https://hal.science/jpa-00247558

Submitted on 1 Jan 1991

HAL is a multi-disciplinary open access archive for the deposit and dissemination of scientific research documents, whether they are published or not. The documents may come from teaching and research institutions in France or abroad, or from public or private research centers.
L'archive ouverte pluridisciplinaire $\mathbf{H A L}$, est destinée au dépôt et à la diffusion de documents scientifiques de niveau recherche, publiés ou non, émanant des établissements d'enseignement et de recherche français ou étrangers, des laboratoires publics ou privés. 


\title{
Smectic order and backbone anisotropy of a side-chain liquid crystalline polymer by Small-Angle Neutron Scattering
}

\author{
L. Norrez ('), G Pépy ( $\left.{ }^{1}\right)$, P. Keller (') and L Benguiguı ( ${ }^{2}$ ) \\ (1) Laboratoıre Léon Brillouın, CEN-Saclay, 91191 Gif-sur-Yvette Cedex, France \\ (2) Solıd State Institute Technıon, 32000 Hasfa, Israel
}

(Recelved 12 November 1990, revised 18 March 1991, accepted 22 March 1991)

\begin{abstract}
We have simultaneously measured, for the first time, the extension of the polymer backbone of a side-chain liquid crystalline polymer and the intensity of the 001 Bragg reflection, which gives the smectic order parameter $\psi$ as a function of temperature in the smectic phase We have qualitatively demonstrated that the more the smectic phase is ordered, the more the polymer backbone is localized between the mesogenic layers It is shown that the Landau theory allows us to relate the radius of gyration parallel to the magnetic field of the polymer backbone to the smectic order parameter We also show that the Renz-Warner theory is surtable at low temperatures
\end{abstract}

\section{Introduction.}

From results obtained by Small-Angle Neutron Scattering [1] and also by X-Ray diffraction [2], it was shown that mesomorphic side-chain polymers become anisotropic in the different mesophases In particular in the smectic A phase, the liquid crystal polymer adopts a more and more oblate shape as soon as the temperature decreases [1c, 1d, le, if] Some polymers reach such an extreme anisotropy in the smectic phase that the extension of the polymer backbone becomes smaller, in the direction of the magnetic field (which is also the direction of the mesogens), than the smectic layer thickness itself [3] It seems highly probable then that the polymer backbone is mainly distributed between the neighbouring mesogenic layers The backbone anisotropy is thus due to an exclusion of the chain from the mesogenic zones. The amount of exclusion that we measure by the anisotropy of the polymer backbone could be directly related to the density profile of the smectic layers, that is to say, the smectic order parameter.

The aim of our experiment is to test whether or not there is a direct correlation between the confinement of the polymer backbone which is given by the component $R_{\|}$of the radius of gyration tensor along the direction perpendicular to the smectic layers and the smectic order parameter which characterizes the probability of finding the mesogens inside the layers We have followed, versus temperature and under the same experimental conditions, the evolution of the 001 Bragg peak intensity (whose square root is proportional to the smectic order parameter) and the evolution of $R_{\mid}$of the polymer backbone in the direction of the magnetic 
field $R_{\|}$is obtained by Small-Angle Neutron Scattering on labelled mixtures [4] of molten liquid crystalline polymers

The experiment was performed with the following sample which is made of a 11 mixture of fully hydrogenated liquid crystal polymer, with the analogous polymer deuterated on the backbone.

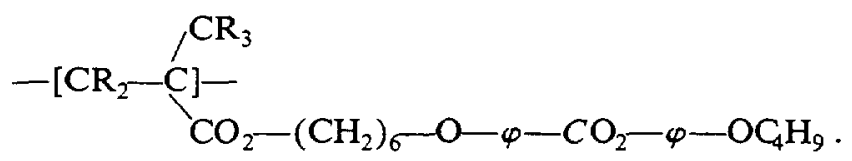

The molecular weights of these compounds are $M_{\mathrm{w}}=250000$ for $\mathrm{R}=\mathrm{H}$ and $M_{\mathrm{w}}=350000$ for $R=D$, and the polydispersities are 22 and 4.4, respectively This system exhibits both nematic and monolayer smectic $A$ phases which appear at $T_{\text {isotropic-nematic }}=108 \mp 1{ }^{\circ} \mathrm{C}$ and $T_{\text {nematic-smectic }}=99 \mp 1{ }^{\circ} \mathrm{C}$ as determined by the optical change of the sample aspect for the isotropic-nematic transition, and the appearance of the Bragg peak for the nematic-smectic transition.

The experimental procedure and the data processing will be described in the next section The third section will be devoted to the results of the measurements of the dimension $R_{\|}^{2}$ and of the smectic order parameter $\psi$ Then, some comments will be made about recent theories, and a first attempt following Landau theory is proposed to fit the results.

\section{Experimental.}

A disk-like cell of $1 \mathrm{~mm}$ thickness, $15 \mathrm{~mm}$ diameter, is filled with the molten mixture and placed in the path of a monochromatic neutron beam (diameter $7 \mathrm{~mm}$ ). A magnetic field of $14 \mathrm{~T}$, perpendicular to the neutron beam, aligns the mesomorphic monodomains. The neutrons scattered by the sample are collected by the plane of a multidetector "PAXY» (Lab Léon Brillouin, CEN-Saclay). The expenmental device PAXY allows us to observe, under the same sample conditions, the small-angle scattering signal [4] and the first Bragg reflection. This is obtained simply by switching the wavelength from $10 \AA$ down to $37 \AA$ keeping the sample-multidetector distance at $18 \mathrm{~m}$. In this way, the scattenng vector $q=\frac{4 \pi}{\lambda} \cdot \sin \frac{\theta}{2}$ (where $\theta$ is the scattering angle) takes on values between $0.01 \AA^{-1}<$ $q<0.11 \AA^{-1}$ when $\lambda=10 \AA$, and $0.03 \AA^{1}<q<03 \AA^{-1}$ when $\lambda=3.7 \AA$

2.1 SMALL-ANGLE NEUTRON SCATTERING (SANS) DATA. - Experimentally [1.e], it appears that the quantity $R_{\|}$is more directly related to the smectic structure than is the quantity $R_{\perp}$ associated with the direction perpendicular to the magnetic field Indeed, in the smectic phase, $R_{\|}$decreases with decreasing temperature in the same way, whatever the compound Note also that the experimental geometry $(\lambda=10 \AA, d=1.8 \mathrm{~m})$ and the high value of $R_{\perp}\left(R_{\perp}>80 \AA\right.$ at low temperatures) do not satisfy the conditions of the Guinier approximation $\left(q_{\perp} \cdot R_{\perp} \leqslant 1\right)$ in the direction perpendicular to the magnetic field and so we cannot measure the evolution of $R_{\perp}$ with enough accuracy Only the thermal variation of $R_{\|}$will be given In the range where the scattered intensity $I^{-1}(q)$ is a linear function of $q^{2}$, we can apply the Guinier approximation and write :

$I^{-1}\left(q_{\|}\right)=I^{-1}(0) \cdot\left(1+q_{\|}^{2} \cdot R_{\|}^{2}\right)$ with $q_{\|} \cdot R_{\|} \leq 1(1)$

where $q_{\|}$denotes the component of the scattered vector along the director, and $R_{\mathrm{I}}$ is as previously defined

22 NEUTRON DIFFRACTION (ND) DATA. - With the experimental conditions which we have adopted $(\lambda=37 \AA, d=18 \mathrm{~m})$, we can observe only the first Bragg reflection on both sides of the beam trap on the PAXY multidetector (see Fig 1). The magnet-sample unit 


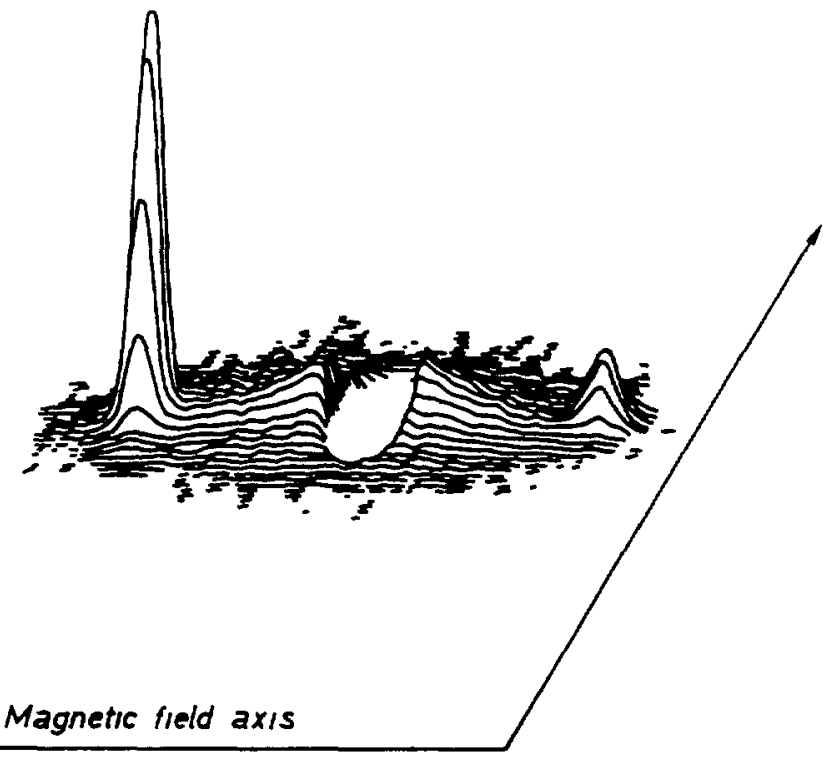

Fig 1 - Scattering pattern obtained in the smectic phase at $485 \mp 05{ }^{\circ} \mathrm{C}$ with a mixture 1:1 of sidechain polymers etther protonated or deuterated on the backbone. The applied magnetic field is horizontal The angle between the mcident beam and the magnetic field is 85 degrees In addition to the 001 Bragg reflections, one notices the Small-Angle Scattering and its anisotropy

rotates about a vertical axis, perpendicular to the neutron beam, in order to search for the maximum Bragg intensity A rocking curve obtained at $60{ }^{\circ} \mathrm{C}$ is shown in figure 2 Two rocking angles $\left(\alpha=5^{\circ}\right.$ and $\alpha=6^{\circ}$ where $\left(\alpha+90^{\circ}\right)$ is the angle between the incident beam and the magnetic field) have been chosen in order to measure the Bragg intensity Each spectrum (containing $128 \times 128$ data points) is then normalized by the spectrum of an incoherent scatterer.

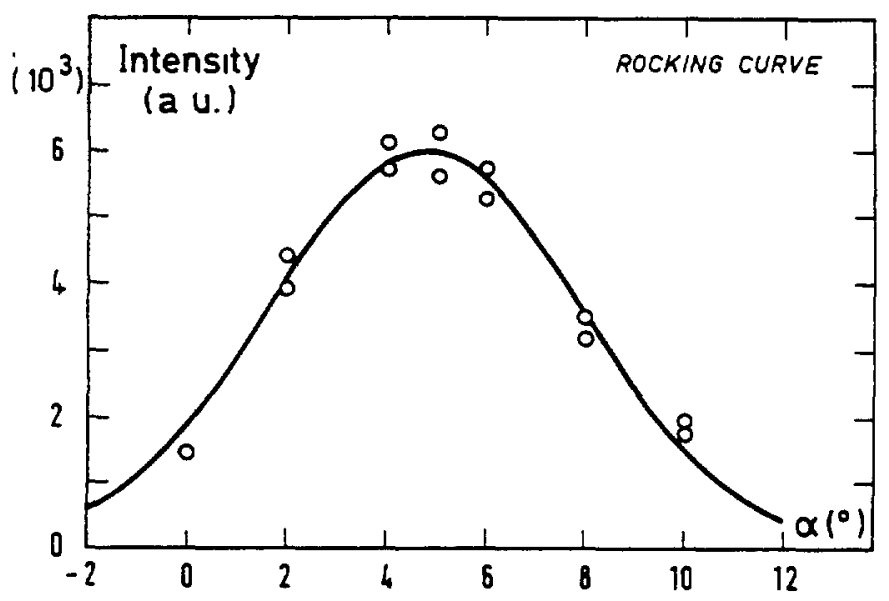

Fig 2 - Rocking curve showing the variation of the integrated intensity of the 001 Bragg peak versus $\alpha$ where $\left(\alpha+90^{\circ}\right)$ is the angle between the incident beam and the direction of the magnetic field 
In order to determine the integrated intensity of the 001 Bragg peak, we model the measured Bragg peak by a two-dimensional Gaussian function (for which we determine the maximum intensity and the full width at half maximum in the two directions parallel and perpendicular to the magnetic field) The small-angle signal (the sample is a mixture of labelled and non-labelled hquid crystal polymers) is modeled by a Lorentzian in agreement with equation (1). We also note that a good fit of the Bragg peak requires that we take into account a small diffuse bump at $001 / 2$ which is fitted by a Lorentzian of weak amplitude This bump could be related to bilayered smectic fluctuations Thus, the determination of the Bragg peak intensity has been extracted from a set of three two-dimensional functions (one gaussian and two Lorentzians)

Figure 3 shows the section of the plane of the multidetector, centered on the direct beam, in the direction parallel to the magnetic field. The continuous line is the fit.

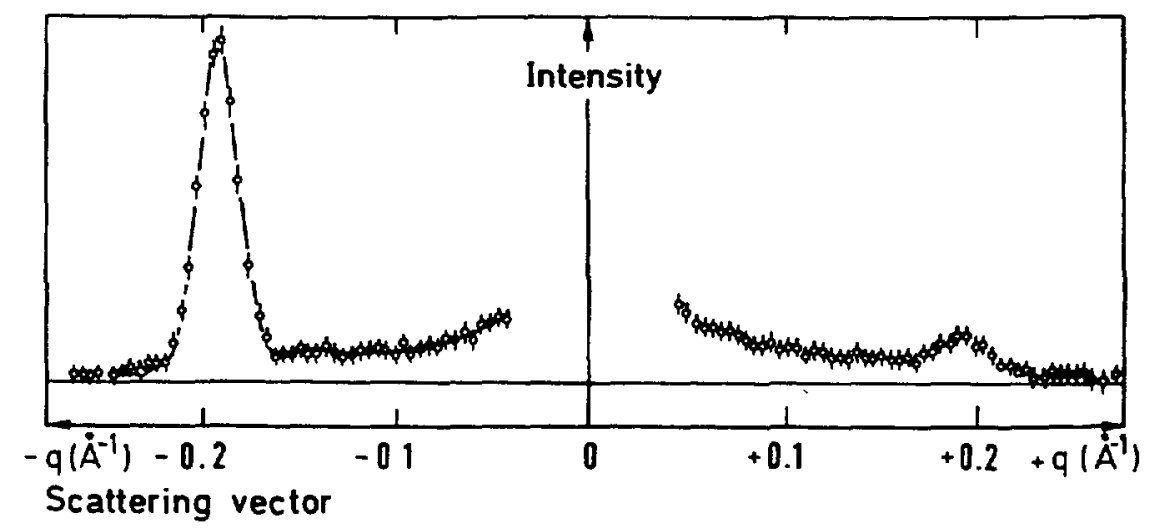

Fig 3 - Section of the plane of the multidetector PAXY centred on the direct beam, in the direction parallel to the magnetıc field $\left(\alpha=5^{\circ}\right)$ The experimental points are represented by the symbol $(0)$. The vertical bars over the symbols are the error bars calculated with the statistical deviation One notices that the intensity of the Bragg peak is not the same at left and at nght since the magnet-sample unit has been rotated about the vertical axis of $5^{\circ}$ in order to bring the smectic layers in position of Bragg reflection Finally, the continuous line corresponds to the fit in the three dimensions

We verified that the sample has reached its thermodynamic equllbrium by repeating the measurements of the Bragg peak intensity at the same temperature The stability of the temperature is about $\mp 05^{\circ} \mathrm{C}$ We also verified the reproducibility of the measurements after a rotation of the magnet-sample unit From these tests, we estimate the error bars on the determination of the Bragg peak intensity to be $10 \%$

2.3 THERMAL PROTOCOL. - The sample (a mixture of fully hydrogenated and partially labelled liquid crystal polymers) was heated in the isotropic phase, for $30 \mathrm{~h}$ in a vacuum oven, in order to eliminate the effect of its thermal history. Then, the sample was slowly cooled until we found, by optical observation, the isotropic-nematic transition. The temperature was then decreased degree by degree, waiting four hours at a given temperature while the small-angle scattering at $\lambda=10 \AA$ was measured. Close to the nematic-smectic transition, we changed the wavelength down $3.7 \AA$ (ND conditions), rotated the magnet-sample unit, and decreased the temperature until we saw the 001 Bragg peak Then, alternate SANS and ND measurements are made at each temperature, down to $25^{\circ} \mathrm{C}$. 


\section{Results.}

31 SMall-ANGle NeUtron SCATtERING STUdy - In figure 4, we display the variation of the component $R_{\|}$, parallel to the magnetic field of the radius of gyration of the polymer backbone, with temperature. As already seen for other liquid crystal polymers studied by SANS, the value of $R_{\|}$is smaller in the mesophases than in the isotropic phase We also notice a strong decrease in $R_{\|}$near the nematic-smectic transition and lasting ten degrees below the transition

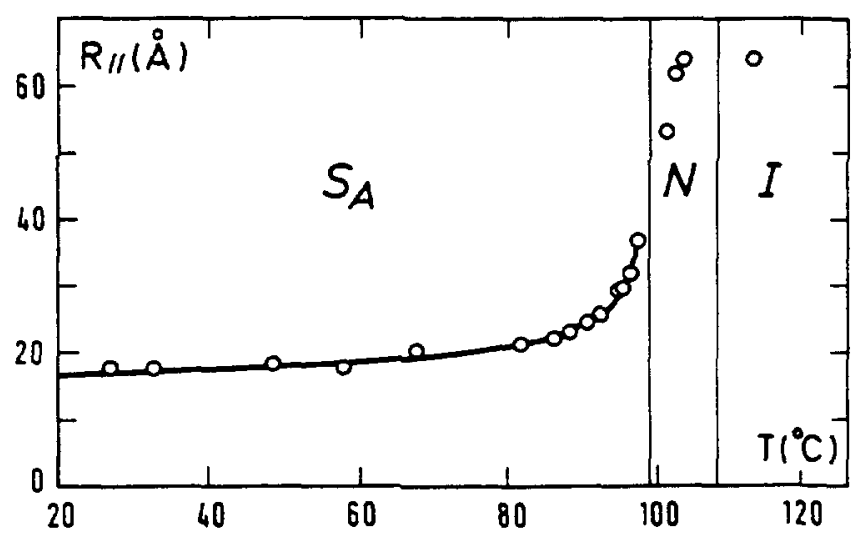

Fig 4 - Variation of the component $R_{\|}$(parallel to the magnetic field) of the radius of gyration of the polymer backbone versus temperature.

We interpret this decrease in $R_{\mathrm{l}}$ by a progressive segregation [1 d,2] of the polymer backbone between the layers of mesogens. Moreover, the decrease of $R_{\|}$in the nematic phase can be explained by the presence of smectic fluctuations which can deform the polymer backbone because the fluctuation range is of the same order as the polymer size.

At the nematic-smectic transition, long-range smectic order is established and the polymer backbone undergoes a sharp contraction in the direction parallel to the mesogens $\left(R_{\mid}\right)$.

After the transition, $R_{\mid}$continues to decrease first rapidly, then more slowly The variation of $R_{\|}$as a function of the temperature can be fitted very well by a power law such as

$$
R_{\|}(\AA)=35 \cdot(98-T)^{-017}=002
$$

Since the polymer chains are not infinite, this law is no longer valid at the nematic-smectic transition (the glass transition gives the limit at low temperatures) This law has no theoretical basis and the exponent can be viewed as an effective exponent Note that the recent theoretical models [5] are also valid far from the temperature transition domain Contrary to what is suggested in [5a], the variation of $R_{\text {l }}$ cannot be modeled by an exponential law $\mathrm{e}^{-E / T}$ where $E$ is a constant and $T$ the temperature Another way to understand the variation of $R_{\|}$, is to assume that the deformation of the polymer backbone is a direct consequence of the smectic order which is better defined when the temperature decreases (the mesogens are then more localized in the layers, independent of the range over which the smectic wave is established at the transition temperature) This is the experimental approach we will develop now, after having first described our diffraction results 
32 NEUTRON DIFFRACTION RESULTS - As explained in section 2, we obtain, by a Gaussian fit, the integrated intensity of the 001 Bragg reflection for two given angles $\left(\alpha=5^{\circ}\right.$ and $\alpha=6^{\circ}$, at different temperatures. The smectic order parameter $\psi$ is proportional to the square root of the intensity of the 001 reflection The smectic order is considered here as a simple sinusoidal distribution of the coherent scattering lengths in the direction perpendicular to the layers This approximation can be justified by the fact that the polymer possesses few reflection orders (001 002 and 003) Moreover, these higher order reflections are always of much weaker intensity than the 001 (according to 3-axis neutron measurements) and are even sometimes missing (as in the case of the 002 reflection of the polymer deuterated on the backbone).

Figure 5 shows the variation of the modulus of the smectic order parameter $\psi$ versus temperature (in the smectic phase). Each temperature in figure 5 is associated with two values of $\psi$ These correspond to the value of $\psi$ for two angles 5 and 6 degrees, for which the Bragg peak intensity is maximum (see $F_{12} 2$ ) The law we deduce for $\psi$ versus temperature is averaged over two angles. The experimental values of figure 5 are well described by a power law. We find:

where

$$
\psi=\psi_{0}+C \cdot\left(973-T^{\circ} \mathrm{C}\right)^{03 \pm 01} \quad \text { (1n arbitrary units) }
$$

$$
\begin{aligned}
\psi_{0} & =0.60 \pm 0.10 \\
C & =1.10 \pm 050
\end{aligned}
$$

It is of importance to outline that the smectic order parameter $\psi$ is measured from a $1: 1$ mixture of hydrogenated polymers and polymers deuterated on the backbone. So, the law given here is a prior only valid for a mixture.

The strong discontinuity in the curve of $\psi$ at the nematic-smectic transition and the nonzero constant $\psi_{0}$ indicate that the nematic-smectic order transition has to be first order. The exponent of the power law $(03 \pm 0.1)$ is of the same magnitude as those obtained in the case

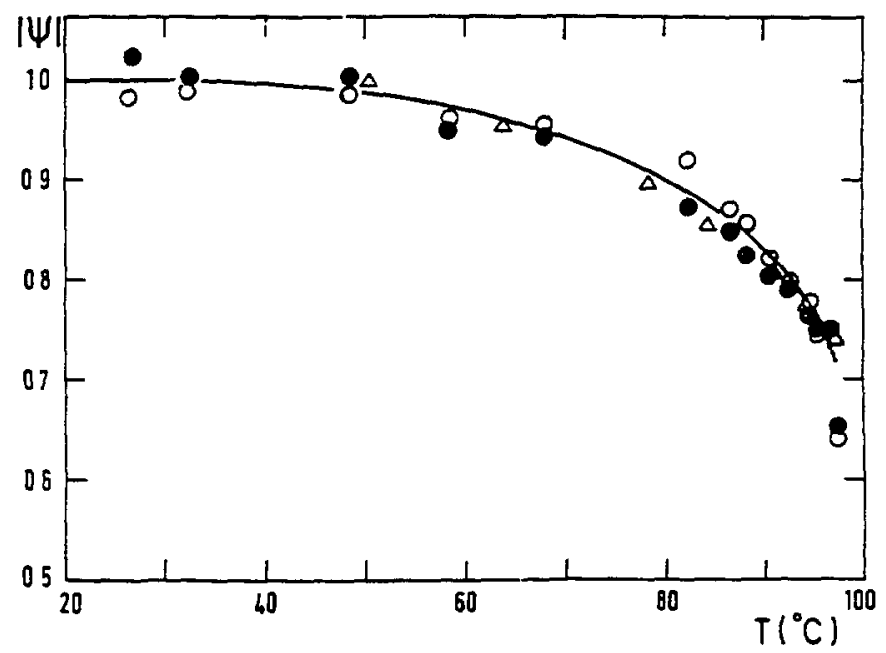

Fig 5 - Variation of the smectic order parameter $\psi$ as a function of the temperature. The black points correspond to the experimental values measured at the angle $\alpha=5^{\circ}(\bullet)$ and the empty points to those measured at $\alpha=6^{\circ}(0)$ The small triangles correspond to the values obtained with the expression (6b) The line is a guide for the eyes 
of small liquid crystal molecules, which are also well described by an effective exponent [6], and is also coherent with a first order transition. Notice that a first order transition has been predicted in the theoretical model of Renz and Warner [5 b] for liquid crystal polymers Within the frame of this theory, the extension $R_{\|}$of the polymer backbone (far from the nematic-smectic transition) must be related to the modulus of the smectic order parameter $\psi$ by an exponential law of form :

$$
R_{\|}^{2} \alpha \exp (-E / k T) \text { where } E \alpha \psi^{1 / 2}
$$

from which

$$
R_{\|}^{2} \alpha \exp \left(-\frac{\psi^{1 / 2}}{A \cdot T}\right)
$$

In order to test this model, we have plotted in figure 6 the logarithmic values of $R_{\mathrm{f}}$ as a function of the square root of $\psi$ divided by the temperature. From figure 6, one can see that the model is not valid close to the nematic-smectic transition (up to $5{ }^{\circ} \mathrm{C}$ after the transition) But, at lower temperatures, a linear domain can be observed in agreement with the assumption of Renz and Warner We determine the constant of the power law to be $A=13 \pm 01$

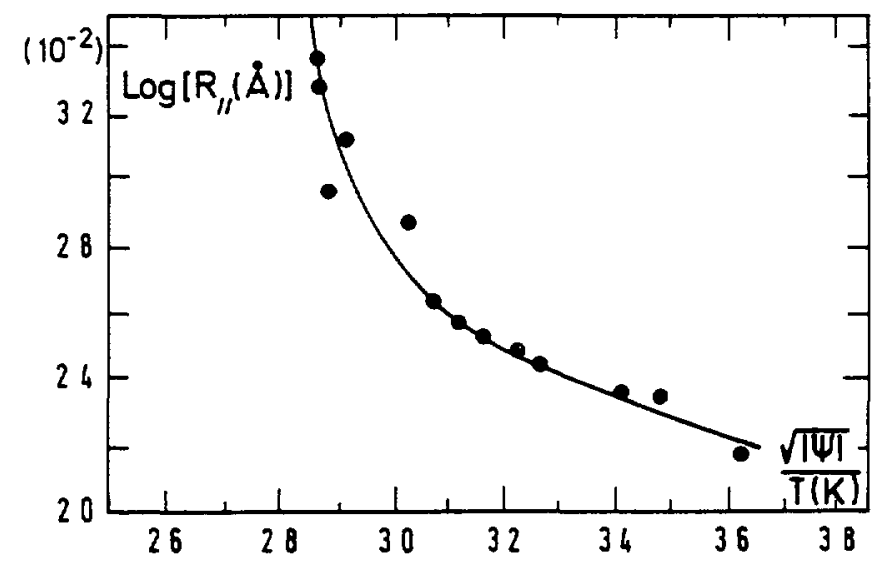

Fig $6-\log \left(R_{1}\right)$ versus the square root of $\psi$ measured at $\alpha=5^{\circ}$ and divided by the temperature (K)

\section{Application of the Landau theory.}

The component $R_{\mathbb{l}}$ of the radius of gyration of the backbone can be related to the smectic order parameter using the Landau theory This phenomenological approach has the advantage of being able to fit the experimental results over the entıre range of temperature, even close to the nematic-smectic transition, in contrast with the assumptions of the theories described above. The liquid crystal polymer can be described by three order parameters the usual nematic order parameter $\left(S_{\mathrm{N}}\right)$, the order parameter of the backbone $\left(S_{\mathrm{B}}\right)$ and the modulus of the order parameter of the smectic phase $\psi$ (see Ref [5.b] for a precise definition of $S_{\mathrm{N}}$ and $S_{\mathrm{B}}$ ) Following Renz [7], in the case of an oblate backbone shape (as in the present case), one has

$$
S_{\mathrm{B}}=\left(\frac{R_{\|}}{R_{\mathrm{1SO}}}-1\right) / 2
$$


In the nematic phase as well as in the smectic phase, we have : $S_{\mathrm{N}} \neq 0$ From the inspection of the X-Ray diffraction patterns at large angles [8], one can see that $S_{\mathrm{N}}$ saturates very quickly as soon as the system is in the nematic phase, and does not present a significant change at the nematic-smectic transition. On the other hand, $S_{\mathrm{B}}$ (through $R_{\mathbb{l}}$ ) exhibits a drastic increase (in absolute value) when passing to the smectic phase. This implies that $S_{\mathrm{B}}$ is strongly coupled to $\psi$ but weakly to $S_{\mathrm{N}}$

Based on the above remarks, we shall make the following assumption we shall consıder only the nematic-smectic A transition and use only two order parameters $\psi$ and $S_{\mathrm{B}} \psi$ is taken equal to the square root of the $001 \mathrm{Bragg}$ intensity; it is a quantity proportional to the smectic order parameter $S_{\mathrm{B}}$ characterzes the backbone ordering and is taken equal to $\left(R_{\|}-R_{\mathrm{iso}}\right) / R_{\text {sso- }}$. We can now write the free energy as a function of $\psi$ and $S_{\mathrm{B}}$.

$$
\begin{aligned}
G=1 / 2 \alpha \cdot S_{\mathrm{B}}^{2}+1 / 3 \beta \cdot S_{\mathrm{B}}^{3}+1 / 2 A \cdot|\psi|^{2}+1 / 4 B \cdot|\psi|^{4}+ \\
\quad+1 / 6 C \cdot|\psi|^{6}+K S_{\mathrm{B}} \cdot|\psi|^{2} .
\end{aligned}
$$

$S_{\mathrm{B}}$ has the same symmetry properties as a nematic order parameter and consequently [9] odd powers of $S_{\mathrm{B}}$ have to be included in $G$. In (3), only $A$ is strongly temperature dependent and can be negative or positive 1.e $A=A_{0}\left(T-T_{0}\right)$ with $A_{0}>0$ Since the polymer backbone cannot order by itself, $\alpha$ is temperature independent Minimizing (3) relatively to $S_{\mathrm{B}}$ and $\psi$ gives :

$$
\begin{aligned}
& \frac{\partial G}{\partial S_{\mathrm{B}}}=\alpha \cdot S_{\mathrm{B}}+\beta \cdot S_{\mathrm{B}}^{2}+K \cdot|\psi|^{2} \equiv 0 \\
& \frac{\partial G}{\partial|\psi|}=A \cdot|\psi|+B \cdot|\psi|^{3}+C \cdot|\psi|^{5}+2 K \cdot S_{\mathrm{B}} \cdot|\psi| \equiv 0
\end{aligned}
$$

These equations admit the trivial solution $S_{\mathrm{B}}=0,|\psi|=0$ which corresponds to the nematic phase. The other solutions $S_{\mathrm{B}} \neq 0$ with $|\psi| \neq 0$, must check the following relations where the values of the coefficients are expermentally determined:

$$
\begin{aligned}
|\psi|^{2} & =-021 S_{\mathrm{B}}-15 S_{\mathrm{B}}^{2} \\
\left(T_{0}-T\right) & =-84|\psi|^{2}+144|\psi|^{4}-024 S_{\mathrm{B}}
\end{aligned}
$$

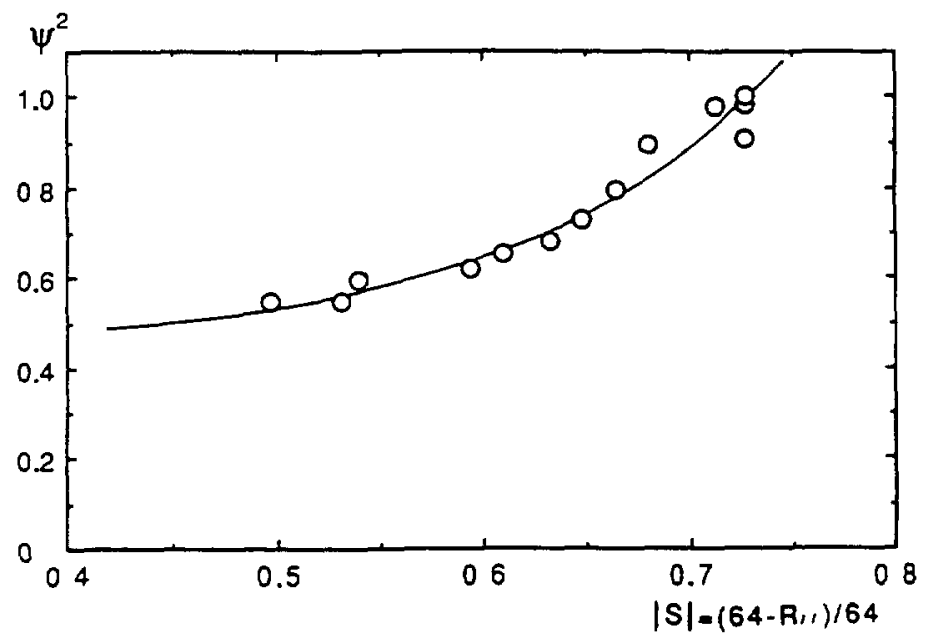

Fig 7 - Square of the modulus of the smectic order parameter $\psi$ versus $|S|$ the modulus of the order parameter of the polymer backbone The points are the experimental values and the continuous line is the fit by the equation (6a) 
where

$$
T_{0}=98^{\circ} \mathrm{C}
$$

In figures 7 and 5, we show the fits of the expermental results with equations (6a) and (6b) The agreement is good, glving us confidence in our choice of the free energy We found that $B<0$, which indicates a first order transition as suggested by the behaviour of $\psi(T)$, $R_{\|}(T) K$ is positive and since $S_{\mathrm{B}}<0$, the coupling term $K S_{\mathrm{B}}|\psi|^{2}$ is negative and this gives a negative contribution to the free energy of the ordered phase, as expected.

In conclusion, equation (3) for the free energy $G$ seems to give a good approach with which to fit the experimental results in the special case of a 1:1 mixture of hydrogenated polymers and polymers deuterated in the backbone. It would also be of interest to know if the same result can be obtained with the pure polymer (all hydrogenated sample or sample with deuterated polymer), in order to know if the intensity of the Bragg peak follows the same evolution versus temperature whatever the labelling.

\section{Conclusions.}

For the first tıme the evolution of the modulus of the smectic order parameter of a mixture H/D of liquid crystal polymers has been measured as a function of the temperature. Moreover, this has been done with a simultaneous measurement of the component $R_{\|}$parallel to the magnetic field of the radius of gyration of the polymer backbone This experiment clearly reveals a parallel between the thermal behaviour of the smectic order parameter $\psi$, which increases with that of $R_{\|}$, which decreases, when the temperature is reduced. The variation of $\psi(T)$ indicates that the nematic-smectic transition of the $\mathrm{H} / \mathrm{D}$ mixture is first order. The effective exponent $(\alpha=03 \pm 01)$, which governs the power law followed by $\psi$, is of the same size as those found for liquid crystals composed of small molecules The question is now to know if the same law can be obtained for each isotopic species, that is to say, whether the local order depends on the site along the mesogens Future experiments with the pure polymers are on the way to measure Bragg intensity versus temperature

Moreover, one has shown that the Renz-Warner theory [5b] can be applied in order to fit those experimental points which are not (as was assumed by the authors) near the nematicsmectic transition (up to $5{ }^{\circ} \mathrm{C}$ below the transition) We also show that the results can be well analysed following the Landau theory

\section{Acknowledgements.}

The authors express their gratitude to Professor M Lambert for her critically reading of this manuscript as well as her helpful suggestions, Dr. A Brulet for the modification of the experimental device which has allowed us to carry out the experiment and $\mathrm{P} M$ Gehring for useful remarks concerning the manuscript

\section{References}

[1] a) Kirste R G, OHM H G, Makromol Chem Raptd Commun 6 (1985) 179

b) Keller P, Carvalho B, Cotron J P, Lambert M, Moussa F, Pepy G, $J$ Phys Lett 46 (1985) 1065

c) Moussa F, Cotton J P, Hardouin F, Keller P, Lambert M, Pepy G, J Phys France 48 (1987)

d) Notrez L., Keller P., Davidson P. Hardouin F, Cotton J P , J. Phys France 49 (1988) 1993

e) NoIrez L, Thesis Orsay 1989 (avalable at the Lab Léon Brillouin). 
f) Noirez L, Moussa F., Cotton J P, Keller P, Pepy G, J. Stat Phys 62 (1991) 991.

g) Kunchenko A B, SVetogorsky D. A, J. Phys 47 (1986) 2015

h) Kalus J., Kostromin S G, Shibaev V P., Kunchenko A B., Ostanevich $Y$ M, SVETOGORSKY D A, Mol Cryst Liq Cryst 155 (1988) 347

[2] a) Davidson P, Keller P, Levelut A M, J Phys France 46 (1985) 939

b) Davidson P, Levelut A M., AChard M. F., Hardouin F., Liquid Crystals 4 (1989) 561

[3] Noirez L, Cotton J P., Hardouin F, Keller P., Moussa F, Pepy G., Strazielle C, Macromolecules 21 (1988) 2889

[4] Cotton J P, Decker D., Benott H, Farnoux B., Higgins J , Jannink G, Des Cloizeaux J , OBER R, PICOT C, Macromolecules 7 (1974) 863

[5] a) RIEger J, $J$ Phys France 49 (1988) 1615.

b) Renz W. WARner M, Phys Rev Lett 56 (1986) 1268.

[6] a) Bartolino R., Durand G, Phys Rev Lett 39 (1977) 1341

b) RICARd L., Prost J, J Phys France C3 40 (1979) 3

[7] RENZ W, in «Polymer Motion in dense systems », D Ruchter and T. Springer Eds. (Springer Verlag) (1987) p 240

[8] Davidson P, private communication

[9] DE GenNes, P G, The Physics of Liquid Crystals (1972) Clarendon Press Oxford. 